\title{
An Overview of Disaster and Emergency Management Systems Models
}

\author{
Dilshad Sarwar \\ Faculty of Business \& Law, Business Systems \& Operations, University of Northampton, \\ Northampton,UK dilshad.sarwar@northampton.ac.uk
}

\begin{abstract}
Emergencies and disasters can cause immense physical, emotional and financial losses to individuals, organisations and larger scale countries. These emergencies and disasters can fundamentally cause casualties and in many cases loss of life. Psychological and financially the impacts on individuals, organisations and countries can be irretrievable due to the impact of the disaster or emergency. Emergency management systems provide a multi-level overview of emergency environments. With a critical examination of current emergency management models, this paper endeavours to review six key framework models for emergency management systems. Bearing the current emergency management models in mind, this paper looks at reviewing the existing six models in order to provide a solution for a more comprehensive emergency management system for implementation. If emergencies are managed effectively these negative impacts can be removed or at least minimised. The paper will evaluate through an extensive literature review, to developing a holistic framework to address the shortfalls of existing models.
\end{abstract}

Keywords: emergency management, crisis management, disaster management model, disaster risk management

\section{INTRODUCTION}

Systems which are used within emergency management are significantly responsible for enabling decisions to be made effectively and thus enabling key resources to be implemented within areas where natural emergencies are prevalent (McCarthy, et al., 2016). The emergency management systems which are used significantly and applied to natural emergencies in undesirable events are required to follow a criterion based approach which have an impact on the critical infrastructure within the environment. When a natural emergency occurs the method used to inform authoritative bodies of the emergency requires a formal alert. This alert system should underpin the key areas where an abnormal situation arises and which is having a direct impact on normal conditions within the environment. The key objective here is to reduce, remove and prevent economical and physical losses through the implementation of an emergency management alert system (Kapucu \& Hu, 2016). Where there is a case of natural emergency occurring the main concern remains to reduce damages and in particular the costs associated with the emergency and implement a defence 
mechanism where required In all cases where there is an emergency an immediate intelligent, informative reaction is necessary (Blaikie, Cannon, Davis, \& Wisner, 1994). This paper highlights key thematic areas; crisis management, emergency management and emergency management. It is necessary here to highlight the constructs which can be applied to natural emergencies which are similar in their destructive nature but which fundamentally have individual characteristics that highlight a variance. Risk management when applied to a natural emergency setting enables the identification, prioritising and the fundamental assessment of natural emergencies. This cycle is therefore followed by focusing particularly on the synchronised and economical use or key resources in order to reduce, monitor and control the likelihood and the effect of natural emergencies, moreover this approach enables the fundamental understanding of the opportunities that are available when a natural emergency occurs.

Where risks are applicable within risks that occur; areas such as failures, natural causes, accidents and natural emergencies in accordance with deliberate attacks; for example terrorist attacks needs to be eliminated or minimised (Li, Li, Liu, Khan, \& Ghani, 2014). Within this natural emergency evaluation crisis management is essentially required in order comprehend what key threats may impair organisations (Karavas, Kyriakarakos, Arvanitis, \& Papadakis, 2015). There are a number of essentials which are key to crisis management, these range from the notion of immediate or direct threats to an organisation, the impact of a natural emergency which causes incredulity and finally a focus on the limited period of making and formulating a decision in the event of a natural emergency. In divergence to the previous focus on risk management which clearly establishes an understanding of risk management and finding alternatives to avoid risks, which in essence focus on ensuring and measuring potential threats and focusing on how to reduce those threats the elements of crisis management endeavour to apportion threats after they have befallen.

It is an area where the control within the greater focus of management aimed at the techniques and skills associated to identifying, understanding, assessing and coping with critical situation, essentially from the time the event initially occurs to the event of actually addressing the impact of the emergency in order to formulate a recovery (Blaikie, Cannon, Davis, \& Wisner, 1994). There are key elements which are outlined within a crisis management model, in essence the diagnosis, and the signals which outline the dangers associated with the emergency. In essence the necessity to change and suggest alternatives are key in developing a turnaround strategy. Further to this the move towards the monitoring and its change process which then necessitates an outcome. Emergency management itself is defined as an area of addressing and avoiding any risks that occur. This requires understanding the fundamental of mitigation, preparedness, response and recovery of the emergency which has occurred (Hughes \& Palen, 2012). It is important to appreciate here that there are very limited essential differences in relation to crisis management and emergency management as a whole (Karavas, Kyriakarakos, Arvanitis, \& Papadakis, 2015).

Emergency management is an area which will be discussed in terms of emergency management models. Emergency management deals directly with the notion of assessing and dealing with a long term focus of organisational management processes which are essentially 
applied to ensure critical assets of an organisation from risks associated with hazards, whereby it is inevitable that catastrophes and emergencies occur. In essence it is important to ensure the continuation within the lifetime of the emergency management system in order to address this problem.

Hazards are categorised as being caused by natural impacts or from human-made impacts (Asghar, Alahakoon, \& Churilov, A Comprehensive Conceptual Modelfor Disaster Management., 2006). The entirety factor applied to strategic management process is clearly divided amongst four key areas namely; risk decrease, resource preparation which are required to respond to hazards and the main elements of damage which are applied to risk limitation, formulating responsive resources to natural emergency, answering to the immediate and definite damage created by hazard, in order to reduce additional damage from occurring, and thus reverting the damage to prior hazard incidents. This thematic area of emergency management is at strategic level therefore considered a strategic process (Kimberly, 2003).

This paper focuses on a thorough literature analysis in order to develop an effective emergency management system which identifies and integrates emergency plans at all key areas of an organisation and thus acknowledging that the initial areas of the organisation have to also consider and are accountable for dealing with the emergency system by having additional resource distribution from the strategic level of the organisation. The requirements to do this are a necessity which are placed on limiting the vulnerabilities in-terms of the socio-economic environment and at the same time addressing other areas which trigger the emergency such as other hazards. The possibility is far greater and intense than other traditional emergency management and therefore enables emergency risk limitation (McCarthy, et al., 2016).

\section{LITERATURE REVIEW}

In this paper a thorough literature review has been undertaken. The literature review highlighted a significant number of models which are fundamental to emergency management systems. Although the focus within this literature review is to address the classical principles of emergency management systems. The literature review does analyse previous papers by (Asghar, Alahakoon, \& Churilove, 2006) however, it is important to emphasise here that this paper focuses more on the holistic development of an emergency management model which addresses the gap in literature found through this literature review. Moreover, research carried out by (Albtoush, Dobrescu, \& Ionescou, 2011) significantly highlights the emergency management models outlined below, from the perspective of implementing intelligent systems concept to emergency management. The authors of this paper however, address these models and signify the difference in-terms of research contributions with a view of addressing natural disasters to develop and formulate an emergency management framework model, in line with developing a structured process associated with any emergency. Contingency planning for any emergency is required as a pre-requisite for the planning of any emergency. Any type of emergency in essence requires a holistic perspective of avoidance, readiness, reaction and recovery. The progress and 
practice of traditional emergency plans have always formulated emergency responses through the most traditional and regimental processes. This paper looks at analysing the current emergency management models which are seen as the underpinning approaches to emergency reactions. The authors believe that it is essential to look at a range of emergency management models in order to develop a framework model which will ensure the identification and implementation of emergency management practices at a holistic level. These models have thus been placed under criterion by means of the Traditional model, Expand and Contract model, Kimberly's model, Tuscaloosa model, Circular model, Manitoba integrated model and the Disaster / Crunch Release model (Cameron, Robinson, \& Yin, 2012). These models will be analysed in terms of their contribution to emergency management requirements. The models discussed within this paper have been discussed and elaborated in the following pieces of research:

Table 1: Existing Emergency Management Models [Asghar, Alahakoon, \& Churilove, 2006; Albtoush, Dobrescu, \& Ionescou, 2011]

\begin{tabular}{|c|c|c|c|c|c|c|c|}
\hline $\begin{array}{l}\text { Emergency } \\
\text { System }\end{array}$ & $\begin{array}{c}\text { Traditional } \\
\text { Model }\end{array}$ & $\begin{array}{c}\text { Two- } \\
\text { Phased } \\
\text { Model }\end{array}$ & $\begin{array}{c}\text { Kimberly } \\
\text { Model }\end{array}$ & $\begin{array}{c}\text { Tuscaloos } \\
\text { a Model }\end{array}$ & $\begin{array}{l}\text { Kelly's } \\
\text { Model }\end{array}$ & $\begin{array}{c}\text { The } \\
\text { Manitoba } \\
\text { Model }\end{array}$ & $\begin{array}{c}\text { The } \\
\text { Emergency } \\
\text { / Relief } \\
\text { Crunch }\end{array}$ \\
\hline $\begin{array}{l}\text { Prediction \& } \\
\text { Detecting }\end{array}$ & $\sqrt{ }$ & $\sqrt{ }$ & & $\sqrt{ }$ & & $\sqrt{ }$ & \\
\hline Alerting & & $\sqrt{ }$ & $\sqrt{ }$ & & $\sqrt{ }$ & & $\sqrt{ }$ \\
\hline Relief & $\sqrt{ }$ & & $\sqrt{ }$ & $\sqrt{ }$ & & $\sqrt{ }$ & $\sqrt{ }$ \\
\hline $\begin{array}{l}\text { Support } \\
\text { after the } \\
\text { emergency } \\
\text { has } \\
\text { occurred }\end{array}$ & & & & & & & \\
\hline
\end{tabular}

The Traditional model has two key stages within it, namely these are Pre-Emergency risk reduction model area and Post-emergency recovery stage (Blaikie, Cannon, Davis, \& Wisner, 1994). The initial phase focuses on the preparation, mitigation and prevention of the emergency (Blaikie, Cannon, Davis, \& Wisner, 1994). The second phase addresses the recovery, response with the onus of development. This is a basic model which does not address the timing of the when the emergency actually happens (Cameron, Robinson, \& Yin, 2012). Moreover, the data incorporation and decision making concept is limited in its achievement (Cameron, Robinson, \& Yin, 2012).

In the Expand and Contract model (Asghar, Alahakoon, \& Churilov, A Comprehensive Conceptual Modelfor Disaster Management., 2006) the processes involved happen concurrently and disregard the consecutive focus of restrictions which were outlined in the Traditional model (Asghar, Alahakoon, \& Churilov, A Comprehensive Conceptual Modelfor Disaster Management., 2006). This model does not address the notion of external and internal elements associated with a hazardous event (Dong, Li, Ota, Yang, \& Zhu, 2014). Moreover, the notional view of any hazardous event occurring other key factors can be seen 
throughout the occurrence that may have effects on the actual hazard occurrence and this model does not allow or follow any consideration should a hazardous event occur. In essence this model is not appropriate for addressing the variety of emergencies which are prevalent (Dong, Li, Ota, Yang, \& Zhu, 2014). When hazardous events occur this model does not effectively contribute to the nature of the occurring emergency.

Kimberly Model and the Tuscaloosa model putrefy the cycle of emergency management in the four key areas: preparation, mitigation, response and recovery (Kimberly, 2003). The key differences outlined in the Kimberly model focused on the mitigation and in essence response based in parallel with the element of recovery outlined on the highest level. The Kimberly model and the Tuscaloosa model necessitate extremely competent employees to ensure that the phases outlined in the models are effectively applied to any emergency occurrence (Cameron, Robinson, \& Yin, 2012). For example emergencies occurring within hospitals, government departments and within educational environments (Hughes \& Palen, 2012).

Kelly's model putrefies the emergency management stages into eight key areas. Kelly initiates a circular model that limits the intensity and understanding of emergencies and further outlines the nonlinear elements of emergency actions (Kelly, 1998). This model enables significant learning to occur from actual emergencies which is a positive focus of the model (Oliver-Smith, Alcantara-Ayala, Burton, \& Lavell, 2016). This model can be seen in the figure below. This model is circular in shape and fundamentally requires the development of a comprehensive and clear database of the impact and internal and external inputs and outputs of the information which requires handling by trained professionals in order to utilise the information and make informed decisions when emergencies occur (Liubartseva, et al., 2016). Moreover, with this model a strong technical infrastructure is required to achieve any form of compliance in achieving reasonable results.

This model for emergency management splits the emergency management into the six key areas: a plan at strategic level, focusing on assessment hazards, risk management, extenuation, and preparedness, monitoring and evaluation - this is related to the Multi-layer Quillinan model (Quillinan, 2009). The model looks at the long term desired state of the emergency management elements for a particular emergency (Blaikie, Cannon, Davis, \& Wisner, 1994). This model is significant within the assessment of hazards, risk management, extenuation and awareness (Quillinan, 2009). The equilibrium amongst flexibility and preparedness is seen as a fundamental benefit of this model (Dong, Li, Ota, Yang, \& Zhu, 2014). Moreover, the infrastructure is required for the Manitoba model to ensure that modifications and adaptations enable the updating of information (Sarwar, Ramachandran, \& Hosseinian-Far, 2017). Again this infrastructure requires well trained and established individuals to operate the functionality of the model. The resources for the model are essentially expensive to implement. Additionally, the cost for this model again is considerably high. The assessment of this model is very much founded on the judgement of users and therefore does not provide a clear and detailed analysis of the effectiveness of the model for all users (Quillinan, 2009). 
The two models for crisis management which focuses on identifying emergencies regardless of the level of emergency in a holistic approach (Quillinan, 2009). The initial model looks at presenting the main stages and points of a crisis within a timeline, which are placed within four core areas (Sylves, 2014). These include the following: the pre-incident stage, event manifestation, support manifestation stage and support occurrence stage (Quillinan, 2009). Each stage identifies the fundamental elements required to evaluate the crisis before the occurrence occurs (Alexander, 2014). During this stage of when the occurrence and the negative elements occur it necessary for feedback to be provided to community bodies (Alexander, 2014). When the completion phase occurs the initial familiarity continues and actions reappear to the elements of the pre-incident stages (Dong, Li, Ota, Yang, \& Zhu, 2014). Some of the stages are prone to overlap and the boundaries applied to the different phases are applied.

Secondly the next model is focused on looking at addressing the technological capabilities which are needed to respond to a crisis (Hughes \& Palen, 2012). This model enables the understanding of evaluating a number of key elements and therefore a clear outline is compulsory requirement by the incident body in the main to appreciate an emergency which has arisen (Kelly, 1998). This model needs resources which are based on information resources, resilient communication when the key information is required from senior level which allow for initial implementation (Cameron, Robinson, \& Yin, 2012). The technologies which are applied to the second model enable an effectual plan at the time of incidence and applied during the development and preparation of the actual future occurrences (OliverSmith, Alcantara-Ayala, Burton, \& Lavell, 2016).

It can be seen that both models are really applied to specific applications regardless of the requirements: This applies to the level of budget, as there is significant high level technology that applies to this model for different areas of geographic places (Quillinan, 2009). The financial focus for the preparation of the emergency happening again is extremely high (Sarwar, Ramachandran, \& Hosseinian-Far, 2017). The initial model is seen to be a time related crisis, in conclusion it can be stated that during this initial period of the time so there is no need for the feedback ( $\mathrm{Li}, \mathrm{Li}$, Liu, Khan, \& Ghani, 2014). It is important to state here that chain management is implemented within the two models, the users of these models look these models which require limitations such as political issues and or cultural environments (Quillinan, 2009). As a crisis happens then that then needs to have a large number of employees who will be the recovery process team (Raju, 2016). However, this may not be appropriate (Simonsohn, Nelson, \& Simmons, 2017).

A more sophisticated model applied to allow complex simulations to occur for crisis management using the specification of the organisational constructs which are focused on (Quillinan, 2009). The three models identified in the part reliance diagram, which also involve land mark ideals. These models enable the emergency management to be outlined (Quillinan, 2009). The operating systems within this model looks at high level to middleware kernel. The subordinate layer implements straightforward elements in relation to the higher layer implementation agent which is area specific workings and policies (Cameron, Robinson, \& Yin, 2012). This model can only be implemented within certain limited regions 
(Karavas, Kyriakarakos, Arvanitis, \& Papadakis, 2015). It is only associated as virtual model therefore it is unclear whether this model will or does provide any real success (Olivares, Canizares, \& Kazerani, 2014).

The Emergency / Crunch Release model is quite an effective model however, the drawbacks with this model are that it focuses too clearly on isolated events which cannot be applied to all emergency types (Cameron, Robinson, \& Yin, 2012). Hence there are short comings in terms of developing a response to natural emergencies effectively (Raju, 2016). The Emergency Crunch Release model focuses on an emergency as it happens and it only initiates this once an emergency occurs and has a direct impact on susceptible people (Oliver-Smith, Alcantara-Ayala, Burton, \& Lavell, 2016). In terms of the Emergency/Crunch model one can only term an emergency has occurred as soon as both points of direct impact on susceptible people actually occurs (Cameron, Robinson, \& Yin, 2012). This model demonstrates that an emergency does not occur regardless of vulnerability as there is no event outlined as a trigger unless impact has occurred (Dong, Li, Ota, Yang, \& Zhu, 2014). This model looks at vulnerabilities and involves the processes associated with removing the emergency (Kimberly, 2003). The positive aspect of this model was to really associate an emergency with the "human elements" associated with the emergency (Oliver-Smith, Alcantara-Ayala, Burton, \& Lavell, 2016). Practitioners associated with emergency management use this model to work on developing an emergency risk management process to deal with vulnerability (Haghighi, Burstein, Zaslavsky, \& Arbon, 2013). The negative aspects of this model are very much in the nature of what it actually focuses on (Bohm, Dietz, \& Benson, 2016). Emergency/crunch release model has a number of negative connotations (Biradar, Hote, \& Saxena, 2016). If an emergency occurs where there is no human involvement then the questions would really focus on how this model could be applied to the different criterion of emergencies (Van De Walle, Turoff, \& Hiltz, 2014). In the previous sections the authors have attempted to address and provide some evaluation of the emergency management models that are discussed in the previous sections (Dong, Li, Ota, Yang, \& Zhu, 2014) . Although the emergency management models outlined above are very apt to use within an emergency, the models to do not signify a holistic approach to emergency management (Raju, 2016). This paper investigates areas for developing and creating a more robust emergency management system for implementation.

\section{METHODOLOGY}

This paper trails the design science methodology which is particularly prevalent to the emergency management aspects of information systems (IS). The design science methodology is essentially very suitable when the research area involves artefacts (Raju \& Ahmed, 2016). Design Science Research Methodology (DSRM) focuses on the three Ps which are namely the key principles, fundamental practices and detailed procedures which necessitate this type of research that focuses on the three key objectives (Venable, Pries-Heje, $\&$ Baskerville, 2016). The authors felt that design science focuses more on and is significantly appropriate to the area of systems and in particular also for organisational and management science which are areas prevalent towards emergency management systems (Simonsohn, Nelson, \& Simmons, 2017). This method is dependable on and does endeavour 
to look at process modelling which emphasises the design science research for this paper (Venable, Pries-Heje, \& Baskerville, 2016). This method is associated with presenting and evaluating design science within the field of systems (Raju \& Ahmed, 2016).

The processes used within this paper include the six key areas that the authors have followed in terms of evaluating the framework models outlined within the paper (Vaishnavi \& Kuechler, 2015). These steps include a clear identification of the frameworks involved within the existing models (Teo, 2016). The overview and definitions associated with the objectives that require an explanation (Venable, Pries-Heje, \& Baskerville, 2016). The framework as a demonstration and an evaluation of the framework which has been carried out and communication of the feedback elements of the framework model (Venable, Pries-Heje, $\&$ Baskerville, 2016). The authors have endeavoured to use design science within this paper through validating and using the methodology for the framework models applied within this paper (Venable, Pries-Heje, \& Baskerville, 2016). The Traditional model, Expand and Contract model, Kimberly's model, Tuscaloosa model, Circular model, Montoba integrated model and the Emergency /Crunch model have been evaluated by the authors using the design science approach (Vaishnavi \& Kuechler, 2015).

The authors believe that using this methodology has enabled them to gain significant overall analysis of the frameworks created and applied to emergency management frameworks (Wieringa, 2014). The authors have used the design science research approach, as it is can provide a clear documented format for analysis and therefore the authors have used it in the analysis and incorporation associated with systems research (Wieringa, 2014). This research approach has two fundamental dimensions, associated with naturalistic versus the artificial evaluation stages (Wieringa, 2014). The authors have also considered validity and reliability of using design science has enabled the authors to consider the framework models more effectively thus allowing triangulation to occur through the analysis of guidelines, evaluation processes and iterations (Simonsohn, Nelson, \& Simmons, 2017). Validity and reliability of this analysis through using this methodology are based on using a set of guidelines to clearly and explicitly focus on improving the overall functionality of the systems under consideration (Wieringa, 2014).

The authors have followed the three cycle approach to design science for this paper (Venable, Pries-Heje, \& Baskerville, 2016). These areas are prevalent to the following three stages environment, design science research and knowledge base (Simonsohn, Nelson, \& Simmons, 2017). The environment focuses on areas such as people, organisational systems, technical systems, problems and opportunities (Simonsohn, Nelson, \& Simmons, 2017). The stages associated with this method has enabled the authors to evaluate the framework models outlined to the framework models (Raju \& Ahmed, 2016). Within the third phase of knowledge base the authors felt using this approach would enable them to look at addressing experiences, expertise and processes that are applicable to the framework models outlined above (Wieringa, 2014).

The authors have used the DSRM approach to identify the problem situation in terms of the existing emergency management models (Raju \& Ahmed, 2016). They have endeavoured to 
look at the design, implementation and evaluation of the existing emergency management models (Venable, Pries-Heje, \& Baskerville, 2016). The authors through the literature review acknowledged that there was a significant area for improvement within the implementation of emergency management systems (Venable, Pries-Heje, \& Baskerville, 2016). The authors have defined clear objectives in order to provide a more holistic framework model covering all aspects of emergency management prevention and support mechanisms (Vaishnavi \& Kuechler, 2015). These areas will be further examined in the further work outlined by the authors. The authors have followed a process of iteration through DSRM throughout this paper (Wieringa, 2014). The specific focus of this paper has been to improve the functional processes for existing emergency management systems. Moreover, the authors believe that by using the DSRM approach is prevalent for this type of study, which is based around systems and systems thinking (Raju \& Ahmed, 2016). The authors further believe that by using this methodology the key three elements of DSRM which include the conceptual principles, design and practice rules can be used effectively to support this study and further study in emergency management systems. Moreover, the design science approach has allowed the authors to a more constructive method for implementation showing a consistent gap in the area of requirements for a more holistic emergency management system. One of the fundamental reasons behind the use of this methodology for this particular research emphasises the implications on the environment, processes and knowledge base and the use of the various cycles of the theories and methods applied to emergency management systems (Raju, 2016).

\section{FINDINGS}

From the extensive literature review carried out by the authors it was evident that there is a need for a more extensive model which will address the requirements of any disaster holistically. The analysis of existing models outlined in the table below suggests that there are shortcomings of the existing models.

Table 2: Analysis of Existing Models and Revised model

\begin{tabular}{|l|c|c|c|c|c|c|c|c|}
\hline $\begin{array}{l}\text { Emergen } \\
\text { System }\end{array}$ & $\begin{array}{c}\text { Tradition } \\
\text { al Model }\end{array}$ & $\begin{array}{c}\text { Two- } \\
\text { Phased } \\
\text { Model }\end{array}$ & $\begin{array}{c}\text { Kimberly } \\
\text { Model }\end{array}$ & $\begin{array}{c}\text { Tuscaloo } \\
\text { sa Model }\end{array}$ & $\begin{array}{c}\text { Kelly's } \\
\text { Model }\end{array}$ & $\begin{array}{c}\text { The } \\
\text { Manitoba } \\
\text { Model }\end{array}$ & $\begin{array}{c}\text { The } \\
\text { Emergen } \\
\text { cy I } \\
\text { Relief } \\
\text { Crunch }\end{array}$ & $\begin{array}{c}\text { Revised } \\
\text { Model }\end{array}$ \\
\hline $\begin{array}{l}\text { Prediction } \\
\text { \& } \\
\text { Detecting }\end{array}$ & $\sqrt{ }$ & $\sqrt{ }$ & & $\sqrt{ }$ & & $\sqrt{ }$ & \\
\hline $\begin{array}{l}\text { Alerting } \\
\text { Relief }\end{array}$ & $\sqrt{ }$ & $\sqrt{ }$ & & & $\sqrt{ }$ & & $\sqrt{ }$ \\
\hline $\begin{array}{l}\text { Support } \\
\text { after the } \\
\text { emergenc } \\
\text { y has } \\
\text { occurred }\end{array}$ & & & & & & & $\sqrt{ }$ \\
\hline
\end{tabular}


Findings suggested that although all the models were significantly focused on emergency management in relation to certain types of emergency situations, all the models analysed did not reflect effectively on the criteria necessary for prediction and detection, alerting and relief. The Traditional model focuses primarily on predicting and detecting an emergency and does not address alerting emergency situations to those who are affected by the emergency disaster which is or has occurred. The traditional model does have some support mechanisms in place for those who are affected with a relief mechanism (Cameron, Robinson, \& Yin, 2012). The Two- Phased model provides no guidance or provision for relief once an emergency has occurred. The Kimberly model is only effective in terms of alerting and providing relief and does not clearly address the prediction and detecting stages of the emergency (Haghighi, Burstein, Zaslavsky, \& Arbon, 2013). As the Traditional model the Tuscaloosa model is again failing to alert to any emergency occurring in which is related to information that the population can benefit from an early warning signs (Bohm, Dietz, \& Benson, 2016). Kelly's model fails to provide any prediction or relief elements which are necessary in any real emergency environment (Alexander, 2014). The Manitoba model similar to the Traditional and Tuscaloosa model focuses on predictions and relief (Alexander, 2014). The Emergency relief crunch model only really signifies a natural disaster if there is some form of human involvement and only operates in terms of alerting and the relief of emergency management (Kapucu \& $\mathrm{Hu}, 2016$ ). The proposed system will encompass prediction and detecting as this in essence will ensure that any emergency will be detected, communities will be alerted and relief will be provided (Mirfenderesk, et al., 2016).

\section{CONCLUSIONS AND FURTHER WORK}

This paper has attempted to provide a literature review and overall analysis of some of the key emergency management models outlined by previous research carried out by (Albtoush, Dobrescu, \& Ionescou, 2011) and (Asghar, Alahakoon, \& Churilove, 2006), in an attempt to address the gap in literature, which focuses primarily at developing an emergency management framework model that takes a whole approach to any emergency regardless of its nature. In essence the paper has outlined and highlighted some of the key elements which have had a significant impact on emergency management systems developments, therefore having an impact on the environments within which they are implemented. The paper has endeavoured to identify the key emergency management models in line with previous research in order to signify and develop an improved emergency management model. Although the models are an analysis the authors believe that there can be further work in order to create an emergency management framework model which looks holistically at emergency prevention, risk assessment, preparedness and mitigation, thus reducing emergencies in a much more comprehensive way, providing a comprehensive approach would involve predictions and detections, alerting and informing communities and finally providing relief to communities and victims of emergency environments that occur.

The suggested model would also provide ongoing support after the emergency has occurred. The proposed model will look at identifying the areas of the population which have been affected and the infrastructure that these communities are living and working in, and in particular developing the infrastructure around the emergency disaster that has impacted on 
the communities involved. The authors have proposed a model which will stipulate that long-term development for sustainability and will require ongoing relief where there will not be a point where recovery will be complete. The proposed model will also focus on enhancing the prevention stages by increasing the detection and preparedness stages of vulnerabilities. Moreover, the authors believe that the proposed model will base recovery on the continuous activities up until the point of the all areas of the infrastructure revert to normality. The authors believe that recovery measures for the new system will enable community bodies to address the short and long term impacts of disaster emergency systems. Additionally, further work will include the development of an emergency management framework model taking into consideration the findings from the extensive literature review.

\section{References}

Adini, B., Ohana, A., Furman, E., Ringel, R., Golan, Y., Fleshler, E., \& Reisner, S. (2016). Learning lessons in emergency management: 4th International Conference on Healthcare Systems Preparedness and Response to Emergencies and Disasters. Disaster and Military Medicine.

Albtoush, R., Dobrescu, R., \& Ionescou, F. (2011). A Hierarchical Model for Emergency Management Systems. University "Politehnica" of Bucharest Scientific Bulletin, Series C: Electrical Engineering, 73(2), 53-62.

Alexander, D. E. (2014). Social media in disaster risk reduction and cirsis management. Science and Engineering Ethics, 717-33.

Asghar, S., Alahakoon, D., \& Churilov, L. (2006). A Comprehensive Conceptual Modelfor Disaster Management. Journal of Humanitarian Assistance.

Asghar, S., Alahakoon, D., \& Churilove, L. (2006). Humanitarian Assistance, 1-15.

Biradar, S., Hote, Y. V., \& Saxena, S. (2016). Reduced-order modeliing of linear time invariant sysems using big bang big crunch optimization and time moment matching method. Applied Mathematical Modelling, 7225-7244.

Blaikie, P., Cannon, T., Davis, B., \& Wisner, B. (1994). Natural Hazards, People's Vulnerability and Disasters. London: Routledge.

Bohm, G. M., Dietz, A., \& Benson, D. (2016). Organizational Resillience. Contemporary Theory and Practice of Organizaations,Part 1: Understanding the Organizations.

Buede, D. M., \& Miller, W. D. (2016). The engineering design of Systems: models and methods.

Buede, D. M., \& Miller, W. D. (2016). The Engineering design of Systems: models and methods. . (J. W. Sons, Ed.) 
Cameron, M. A., Robinson, B., \& Yin, J. (2012). Emergency situation awareness from twitter for crisis management. In Proceedings of the 21st International Conference on World Wide Web, 695-698.

Dong, M., Li, H., Ota, K., Yang, L. T., \& Zhu, H. (2014). Multicloud-based evacuation services for emergency management. IEEE Cloud Computing, 50-59.

Haghighi, P. D., Burstein, F., Zaslavsky, A., \& Arbon, P. (2013). Development and evaluation of ontology for intelligent decision support in medical management for mass gatherings. Decision Support Systems, 1192-1204.

Hughes, A. L., \& Palen, L. (2012). An evolving role of the public information officer: an examination of social media in emergency management. Journal of Homeland Security and Emergency Management, 1-20.

Kapucu, N., \& Hu, Q. (2016). Understanding Multiplexity of collaborative emergency management networks. The American Review of Public Administration, 399-417.

Karavas, C. S., Kyriakarakos, G., Arvanitis, K. G., \& Papadakis, G. (2015). A multi-agent decentralized energy management system based on distributed intelligence for the design and control of autonomous polygeneration microgrids. Energy Conversion and Management, 166-179.

Kelly, C. (1998). Simplifying Disasters: Developing a model for complex Non-Linear Events". International Conference on Disaster Management: Crisis and Opportunity: Hazard Management and Disaster Preparedness in Australia and Pacific Region, 2528.

Kimberly, A. (2003). Disaster Preparedness in Virginia Hospital Centre - "Arlington after September 11 2001". Disaster Management and Response, 80-86.

Li, J., Li, Q., Liu, C., Khan, S. U., \& Ghani, N. (2014). Community-based collaborative information system for emergency management. Computers and Operations research, 116-124.

Liubartseva, S., Pinardi, N., De, D. M., Lecci, R., Turrisi, G., \& Palermo, F. (2016). Decision support system for emergency management of oil spill accidents in the Mediterranean Sea. Natural Hazards and Earth System Sciences, 2009-2020.

McCarthy, N., Neville, K., Pope, A., Gallagher, A., Nussbaumer, A., \& Steiner, C. M. (2016). The Creation of a training model to support decision-making of emergency management practioners: A design research study. Journal of Decision Systems, 558565.

Mirfenderesk, H., Carroll, D., Chong, E., Jafari, A., Hossain, N., van Doom, R., \& Vis, S. (2016). New generation flood forecasting and decision support system for emergency management. Australian Journal of Emergency Management, 31(2), 31. 
Olivares, D. E., Canizares, C. A., \& Kazerani, M. (2014). A centralized energy management sysem for isolated microgrids. IEEE Transactions on smark grid, 1864-1875.

Oliver-Smith, A., Alcantara-Ayala, I., Burton, I., \& Lavell, A. (2016). The social construction of disaster risk: seeking root causes.

Ozdamar, L., \& Ertem, M. A. (2015). Models, solutions and enabling technologies in humanitarian logistics. European Journal of Operational Research, 55-65.

Ozdamar, L., \& Ertem, M. A. (2015). Models, solutions and enabling technologies in humanitarian logistics. European Journal of Operational Research, 55-65.

Quillinan, T. B. (2009). Developing Agent-Based Organisational Models for Crisis Management. Autonomous Agents and Multiagent Systems.

Raju. (2016). Disaster Risk Management in Asia and the Pacific. The Copehagen Journal of Asian Studies., 111-112.

Raju, P., \& Ahmed, V. (2016). Design science methodology for developing a learning object repository for construction. Research Methodology in the Built Environment. A Selection of Case Studies.

Sarwar, D., Ramachandran, M., \& Hosseinian-Far, A. (2017). Disaster Management Systems as an Element of Risk Management for Natural Disaster Systems Using the PESTLE Framework. 11th International Conference on Global Security, Safety and Sustainability, London.

Simonsohn, U., Nelson, L., \& Simmons, J. (2017). Research Methodology, Design and Analysis. Annual Review of Psychology, 1(69).

Sylves, R. (2014). Disaster policy and politics - Emergency management and homeland security.

Teo, T. (2016). An Effective Interdisciplinary Teaching and Learning Methodology through Crossed-Subjects Design Project. GSTF Journal on Education (JEd), 2(3), 1-4.

Vaishnavi, V. K., \& Kuechler, W. (2015). Design science research methods and patterns: innovating information and communication technology. Crc Press.

Van De Walle, B., Turoff, M., \& Hiltz, S. R. (2014). Informaiton Systems for emergency management. Routledge.

Venable, J., Pries-Heje, J., \& Baskerville, R. (2016). A Framework for Evaluation in design science research. European Journal of Information Systems, 77-89.

Wieringa, R. (2014). What Is Design Science? In Design Science Methodology for Information Systems and Software Engineering, 3-11. 